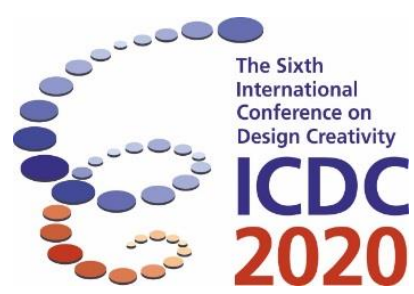

\title{
Sustaining creativity with neuro-cognitive feedback: a preliminary study
}

\author{
Tripp Shealy ${ }^{1}$, John Gero ${ }^{2}$, Julie Milovanovic ${ }^{3}$ and $\mathrm{Mo} \mathrm{Hu}^{1}$ \\ ${ }^{1}$ Departement of Civil and Environmental Engineering, Virginia Tech, Blacksburg, VA, USA \\ ${ }^{2}$ Department of Computer Science and School of Architecture, University of North Carolina \\ at Charlotte, Charlotte, NC, USA \\ ${ }^{3}$ Department of Architecture, Graduate School of Architecture and Ecole Centrale, Nantes, \\ France
}

\begin{abstract}
Ideation is a key phase in engineering design and brainstorming is an established method during ideation. The proposal of ideas tends to peak at the beginning of the brainstorming process and quickly decreases over time. In this preliminary study, we tested an innovative solution to sustain ideation by providing engineering designers feedback about their neuro-cognition. We used a novel neuro-imaging technique called functional near-infrared spectroscopy (fNIRS) to monitor engineering design students during a brainstorming task. Half were given real-time feedback about their brain activation. Our results show that these students applied more cognitive effort in the region of the brain generally associated with memory retrieval and making associations compared to the control group of students that were not provided neuro-feedback. Students that received neuro-feedback also generated significantly more concepts over time and displayed a higher fluency of engineering design solutions.
\end{abstract}

Keywords: neuro-cognitive feedback, fNIRS, design engineering, creativity, brainstorming

\section{Introduction}

A critical step in the engineering design process is ideation (Cross, 1989). Anything that enriches this step in the design process has the potential to improve engineering outcomes that benefit society. Ideation, or idea generation, brings together problem understanding, engineering science, social factors and practical knowledge to develop new concepts and possible solutions (French, 1999). The quality and quantity of ideas generated during ideation informs and even determines the outcome of design (Helm et al., 2016). However, a problem during the ideation process is the production of new ideas tends to peak early in the design process and decline over time (Shealy et al., 2018), which can lead to a fixation effect on early solutions (Gero, 2011).

In this paper, we present an experiment that tests a technique to sustain idea generation by providing engineering designers feedback about their neuro-cognition. Neuro-cognitive feedback has had success in other domains (Hammond, 2011), for example to train musicians on attention and relaxation to improve their performance (Egner \& Gruzelier, 2003) or as a collaborative and social experience for an art performance (Kovacevic et al., 2015). By providing designers neuro-cognitive feedback, we aimed to extend the time and cognitive effort to generate new ideas. 
In the following section, we describe principles and use of one method for ideation, called brainstorming. In section three, the potential of neuro-cognitive feedback is explained in more detail followed by our research question, methodology and results. The results highlight the effects of neuro-cognitive feedback to sustain ideation. The discussion and conclusion provide ground for future research.

\section{Brainstorming: an established method for idea generation}

A large number of techniques are available to assist designers during idea generation (Smith, 1998). Brainstorming is one of these techniques, and it has remained a dominant technique for decades (Isaksen $\&$ Gaulin, 2005). During brainstorming, designers are instructed to imaginatively generate as many ideas as possible while suspending criticism of the ideas being generated (Hernandez et al., 2010). Previously generated ideas are combined to form new ones (Pinsonneault et al., 1999). Only after this process ends should judgement be applied to filter concepts with given parameters (Osborn, 1953).

The cognitive process needed for brainstorming originates in the mental structures that control memory cognition (Potter \& Balthazard, 2004). Brainstorming works by classifying attributes of ideas into shortterm memories, which serve as probes to seek and retrieve matching traces in our long-term memory and transform these memories into new and modified mental schemas (Cross, 2001). To continue to brainstorm requires repeated prompts to memory functions that re-initiate the search process. More mental energy is required for each new search process (Alexiou et al., 2011).

\section{Developing tools to sustain creativity: the potential of neuro-feedback}

Prior research has shown that the cognitive search process during ideation tends to decay over time (Viswanathan, 2017), resulting in many solutions early in the process and fewer solutions later in the process (Helquist et al., 2007; Howard et al., 2008). This reduction in idea generation over time might be because the prior products of memory search that lead to the earlier ideas begin to interfere with the generation of new ideas (Ditta \& Storm, 2017; George et al., 2019; Storm \& Hickman, 2015). The decay of ideas may also be due to a resource constraint (Shealy \& Hu, 2017). Devoting new cognitive resources to fewer ideas eventually becomes ineffective and thus designers stop expending new cognitive resources (Alexiou et al., 2011; Shealy et al., 2018). In either explanation, the number of new ideas being retrieved or generated during brainstorming decreases over time (Helquist et al., 2007; Howard et al., 2008). The decrease of idea generation over time can induce a fixation effect on an early solution (Gero, 2011), thus reduce the solution space explored by designers.

To address the issue of a reduction in idea generation over time and to sustain creativity during brainstorming, we explored the potential of neuro-cognitive feedback. Neuro-cognitive feedback provides a biofeedback on participants' brain activity in order to encourage self-regulation. Studies in fields unrelated to engineering design have shown that self-regulation of specific brain activity can be learned with neuro-cognitive feedback (Egner \& Gruzelier, 2003; Kovacevic et al., 2015). Neurocognitive feedback interventions can occur in-situ and are inexpensive compared to the actual cost of engineering design. Other interventions, such as human interactions, exist to sustain concept generation but neuro-cognitive information provides customized feedback. This customized feedback may help teach self-regulation. The aim of this study was to establish grounds for future development of a neurocognitive feedback tool that can help design engineers better understand their design patterns of performance and improve their mental ability to quickly adjust their cognitive approach during design.

\section{Research question}

Our research question is what are the effects of neuro-cognitive feedback on idea generation during a brainstorming task? To answer this research question, we measured the number of ideas generated during a brainstorming session, the fluency of design ideas by analyzing the time span between ideas, and the cortical activation in the regions of the brain associated with concept generation tasks. The purpose of measuring change in cortical activation was to observe the neurological effect of feedback on engineering design. 


\section{Methodology}

To explore the effects of neuro-cognitive feedback on idea generation, we asked ten graduate engineering students from Virginia Tech (all male and right-handed) to develop a range of possible solutions using brainstorming for a design task about the first/last mile mobility problem. The first/last mile problem is about transporting people between mass transit stops and their residential dwellings (Tilahun et al., 2016). Students were asked to verbally describe their solutions. They were told the goal of the design task was to develop as many solutions as possible. They were given no time limit to complete the task. To compare the difference in the number of ideas between the control and experimental group we used an independent t-test.

Before the task began, all ten graduate engineering students were outfitted with our neuroimaging instrument that measures change in oxygenated blood in the prefrontal cortex (PFC). We provided half of the students real-time feedback about their cognitive activation in their prefrontal cortex. The purpose of providing this feedback was to help them become more self-aware of their cognitive activation patterns and help them sustain activation. Participants were instructed to look at their feedback and try to sustain activation to keep receiving feedback as red on the heat map of cortical activation in their PFC. The aggregated change in oxy-hemoglobin across their PFC is represented on the heat map with specific channels, shown in Figure 1. A running average of 30 seconds was used to produce the heat map, where red means high activation, green neutral, and blue negative. The 30 second average represents the time of two full hemodynamic responses from subjects. A hemodynamic response is the time from rest to peak and back to rest as a result from stimuli, which is generally 15 seconds from the onset of the stimulus. The heat map was generated using Shimadzu's LIGHTNIRS system.

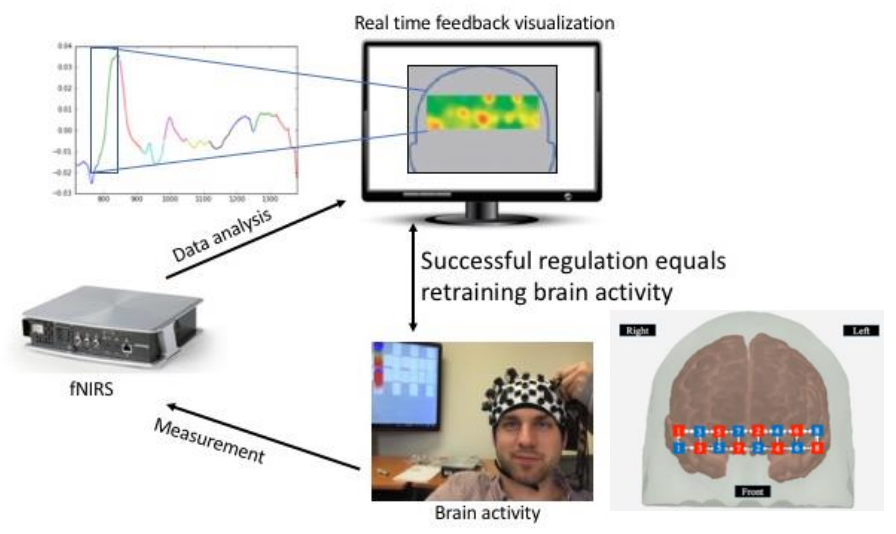

Figure 1. fNIRS neuro-cognitive feedback device

Students decided when they were finished with the task, so their time to complete the task was grouped into ten equal segments or deciles because of the variability in time between students. Participants' cognitive activation was averaged together for these segments. This segmenting technique is similar to previous engineering design cognition studies (Gero et al., 2013). Segmenting normalized time across subjects and allowed us to average patterns of cognitive activation across time.

\section{Tool to measure neurocognition}

We used functional near-infrared spectroscopy (fNIRS) to provide neuro-cognitive feedback. fNIRS provides higher spatial resolution than electroencephalography (EEG) and a more realistic setting than functional magnetic resonance imaging (fMRI) (Shealy \& Hu, 2017). fNIRS is also easier to prepare for neuro-cognitive feedback trials compared to EEG and allows subjects to sit in a relatively naturalistic setting compared to fMRI. Data collection with fMRI is constraining because the scanner encloses participants. The recent development of portable fNIRS devices also makes the potential for ecological validity greater than fMRI. fNIRS works similarly to fMRI but it emits a near-infrared light into the human cortex, and refracted light that is not absorbed is detected by sensors (Ferrari \& Quaresima, 2012). The change in light absorption indicates change in oxy- and deoxy-hemoglobin. 
The region of interest for measuring change in oxy-hemoglobin is the prefrontal cortex (PFC) and its sub-regions including dorsolateral PFC (dlPFC) and the medial PFC (mPFC). These sub-regions within the PFC are the focus of most cognitive studies about design (Shealy et al., 2017), decision making (Hu \& Shealy, 2019), and planning because of their associated cognitive function. The dlPFC is associated with cognitive flexibility, working memory, and abstract reasoning (Pochon et al., 2002; Soltanlou et al., 2018). The mPFC is a critical region for memory retrieval. The data we used to provide neurocognitive feedback and the data we used for analysis was the change in oxy-hemoglobin over time in the PFC and these subregions. We used oxy-hemoglobin instead of deoxy-hemoglobin or total hemoglobin because it is the most sensitive signal to changes in cerebral blood flow. The arrangement of sensors and detectors is illustrated in Figure 1.

\section{Results}

\section{Creativity and fluency of design ideation}

The students who received the neuro-feedback produced more ideas ( 7.8 ideas on average) compared to the control group (3.8 ideas on average). The time participants spent brainstorming was not significantly different $(p=.76)$ between our two groups. Students with the neuro-cognitive feedback spent less time brainstorming ( $223 \mathrm{sec}$. on average) than the control group (242 sec. on average). A t-test between our groups shows that the time between ideas (fluency) is significantly less $(p=.04)$ for the experimental group ( 29 seconds between ideas on average) compared to the control group ( 65 seconds).

Table 1. Design metrics

\begin{tabular}{lcc} 
& Control (SD) & Neuro-feedback (SD) \\
\hline \hline Number of ideas & $3.8(1.1)$ & $7.8(4.2)$ \\
\hline Total time in seconds & $242(132)$ & $223(40)$ \\
\hline Fluency, time between concepts in seconds & 65 & 29 \\
\hline \hline
\end{tabular}

\section{Neurophysiological activation in the prefrontal cortex (PFC)}

To explore students' neuro-cognitive activation across time, we looked at the interpolation of the average of oxygenated blood in the pre-frontal cortex for each decile for our control group (no neurofeedback) and experimental group (neuro-feedback). For our control group, our analysis shows a decay in brain activation during brainstorming, Figure 2. The slope of the linear fit is negative, which indicates a decrease in neuro-cognitive activation over time. When students were given real-time feedback about their brain activation, they applied more cognitive effort in the pre-frontal cortex, Figure 3. The neurocognitive feedback intervention reversed the decay of activation during concept generation as the slope of the linear fit is positive.

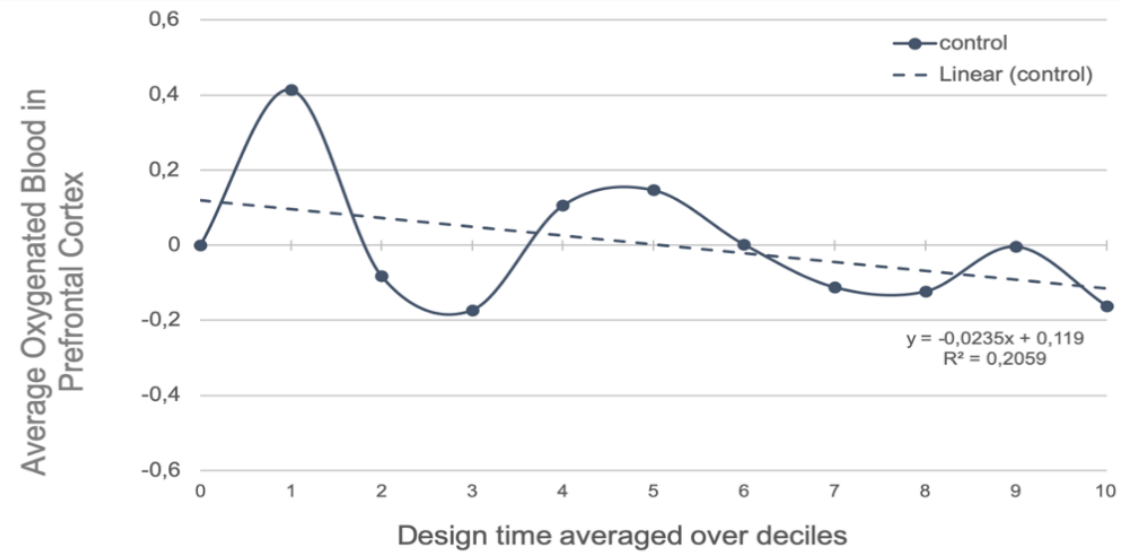

Figure 2. Average of $\mathrm{Oxy}-\mathrm{Hb}(\mathrm{uM})$ in the $\mathrm{PFC}$ across time for control group 


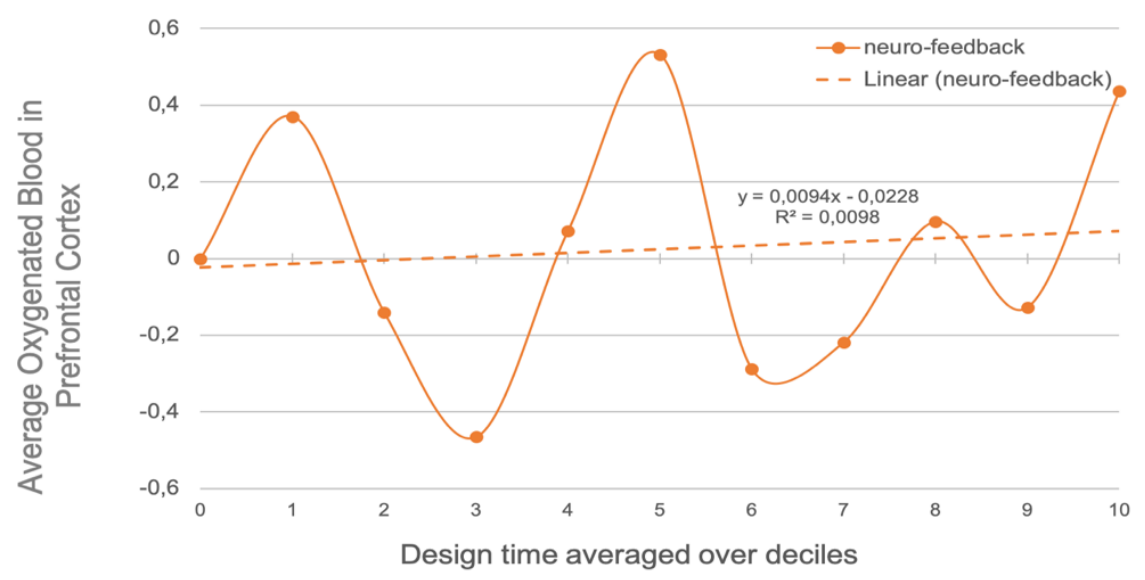

Figure 3. Average of $\mathrm{Oxy}-\mathrm{Hb}(\mathrm{uM})$ in the PFC across time for neuro-cognitive feedback group

Another finding is the similarity of the evolution of the cortical activation over time that follows a wavy curve, Figure 4. This phenomenon appears in both groups and illustrates an alternation of activation and deactivation that might account for a cycle in the idea generation process. We also note that for our experimental group, the amplitude of the wave is higher than for our control group. Moreover, it remains the same across time when neuro-cognitive feedback is given, whereas without this feedback, the amplitude drops as the ideation session reaches its end.

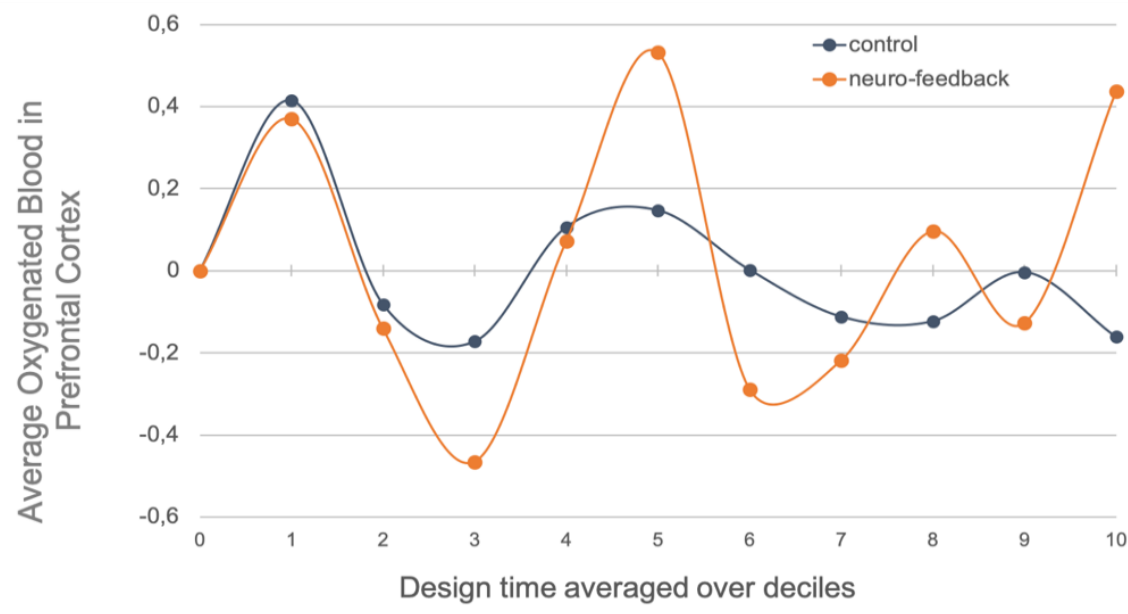

Figure 4. Average of $\mathrm{Oxy}-\mathrm{Hb}(\mathrm{uM})$ in the PFC across time for both groups

\section{Activation in subregions of the PFC}

The average oxygenated blood (oxy-Hb) in the right and left hemispheres of the PFC was compared between the control and experimental group. Oxy-Hb was consistently greater in the experimental group in all channels of the PFC compared to the control group except in channels one and two, along the right dlPFC. The right dIPFC is known to contribute to improvisation (Rosen et al., 2016). The largest difference in oxy-Hb between the experimental and control group occurred in the right mPFC. This region is connected with association tasks (Bhattacharya \& Petsche, 2002) and is observed to play a role in the retrieval of "remote" memories (Euston et al., 2012). The average oxy-Hb is positive in the neurofeedback group and negative in the control group, illustrated in Figure 5(a). The differences between groups in the mPFC become even more pronounced later in the brainstorming process. The difference in oxy-Hb increases by over 250 percent when isolating activation in the second half of the brainstorming process, in deciles 6-10, illustrated in Figure 5(b). 


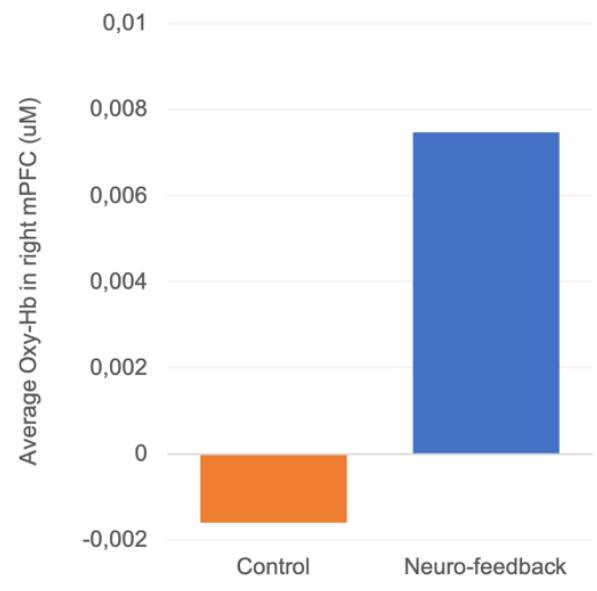

(a)

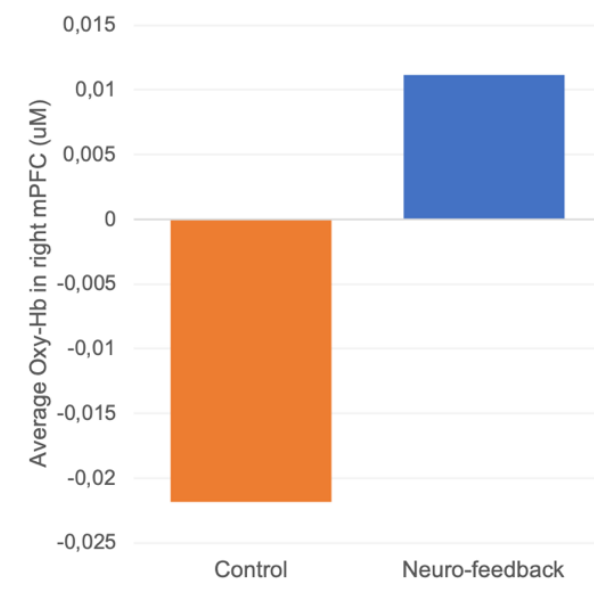

(b)

Figure 5. Average of oxygenated blood (uM) in the right mPFC for each group for (a) entire session (all deciles), (b) for the last deciles (6 to 10)

\section{Discussion and Conclusion}

Our results show a positive impact of the neuro-cognitive feedback on students' creative behavior during brainstorming. Neuro-cognitive feedback did not extend the time participants spent brainstorming, but rather helped them become more efficient during the task as there was a significant improvement in the fluency of design ideation. Across time, participants with the neuro-cognitive feedback succeeded in maintaining cortical activation in their PFC, and this coheres with our findings on design fluency. While not all neurological changes have a causal relationship with cognition and behavior (Fishburn et al., 2014), an association between the number of ideas proposed and the increase of oxygenated blood in the PFC seems evident. Student designers in the experimental group exhibited more oxygenated blood in all regions of the PFC, except two channels in the right dlPFC. The PFC has known associations with improvisation (Rosen et al., 2016). Perhaps designers in the experimental group exhibited more directed attention and fixation on the neuro-cognitive feedback and this limited the cognitive resources to this region. The largest positive difference in activation occurs in the right medial PFC, a region associated with memory retrieval and making associations. The difference is most pronounced later in the brainstorming process. This might suggest designers continued to retrieve memories to sustain neurocognitive activation, which is supported by the increase in the number of ideas from designers in the experimental group.

These results should be interpreted with some caution because of our limited sample size. A larger sample and multiple additional studies are needed before any type of generalization. Future work should investigate the relationship between region dominance (Shealy \& Gero, 2019), could compare patterns of activation using network analysis (Shealy et al., 2018) and evaluate the effect of neuro-feedback on the quality of ideas generated (Shah et al., 2003). With more participants, future studies can begin to use more advanced statistical methods and prediction models to explain the relationship between increased oxy-Hb in the right $\mathrm{mPFC}$ and design fluency and creativity. While this project is focused on ideation, similar opportunities for improved performance during multiple phases of design are also possible. For example, the results demonstrate the potential for an entirely new class of engineering design tools. While much current research advances computers to replace humans, these findings suggest a future where neuro-cognitive feedback aids, rather than replaces, human cognition. This research and future studies can also be directly integrated into engineering education to support selfregulated learning and provide new types of active learning pedagogy in engineering since this feedback is immediate. 


\section{References}

Alexiou, K., Zamenopoulos, T., \& Gilbert, S. (2011). Imaging the designing brain: A neurocognitive exploration of design thinking. In J. S. Gero (Ed.), Design Computing and Cognition '10 (pp. 489-504). https://doi.org/10.1007/978-94-007-0510-4_26

Bhattacharya, J., \& Petsche, H. (2002). Shadows of artistry: Cortical synchrony during perception and imagery of visual art. Brain Research. Cognitive Brain Research, 13(2), 179-186.

Cakir, M. P., Cakar, T., Giriken, Y., \& Demirciolu, A. K. (2016). Neural correlates of purchasing decisions in an ecologically plausible shopping scenario with mobile fnir technology. Lecture Notes in Computer Science (Including Subseries Lecture Notes in Artificial Intelligence and Lecture Notes in Bioinformatics), 9743, 135146. http://dx.doi.org/10.1007/978-3-319-39955-3_13

Cross, N. (2001). Design cognition: Results from protocol and other empirical studies of design activity. In Design knowing and learning: Cognition in design education (pp. 79-103). Elsevier.

Cross, N. (1989). Engineering Design Methods. Wiley.

Ditta, A. S., \& Storm, B. C. (2017). That's a good idea, but let's keep thinking! Can we prevent our initial ideas from being forgotten as a consequence of thinking of new ideas? Psychological Research, 81(3), 678-689. https://doi.org/10.1007/s00426-016-0773-2

Egner, T., \& Gruzelier, J. H. (2003). Ecological validity of neurofeedback: Modulation of slow wave EEG enhances musical performance. NeuroReport, 14(9), 1221.

Euston, D. R., Gruber, A. J., \& McNaughton, B. L. (2012). The role of medial prefrontal cortex in memory and decision making. Neuron, 76(6), 1057-1070. https://doi.org/10.1016/j.neuron.2012.12.002

Ferrari, M., \& Quaresima, V. (2012). A brief review on the history of human functional near-infrared spectroscopy (fNIRS) development and fields of application. NeuroImage, 63(2), 921-935. https://doi.org/10.1016/j.neuroimage.2012.03.049

Fishburn, F. A., Norr, M. E., Medvedev, A. V., \& Vaidya, C. J. (2014). Sensitivity of fNIRS to cognitive state and load. Frontiers in Human Neuroscience, 8, 76. https://doi.org/10.3389/fnhum.2014.00076

French, J. M. (1999). Conceptual Design for Engineers | Michael J. French | Springer (3rd ed).

George, T., Wiley, J., Koppel, R. H., \& Storm, B. C. (2019). Constraining or constructive? The efects of examples on idea novelty. The Journal of Creative Behavior, 53(3), 396-403. https://doi.org/10.1002/jocb.178

Gero, J. S. (2011). Fixation and commitment while designing and its measurement. The Journal of Creative Behavior, 45(2), 108-115.

Gero, J. S., Jiang, H., \& Williams, C. B. (2013). Design cognition differences when using unstructured, partially structured, and structured concept generation creativity techniques. International Journal of Design Creativity and Innovation, 1(4), 196-214. https://doi.org/10.1080/21650349.2013.801760

Hammond, D. C. (2011). What is neurofeedback: An update. Journal of Neurotherapy, 15(4), 305-336. https://doi.org/10.1080/10874208.2011.623090

Helm, K., Jablokow, K., McKilligan, S., Daly, S., \& Silk, E. (2016). Evaluating the impacts of different interventions on quality in concept generation. 123rd ASEE Annual Conference and Exposition, New Orleans, USA.

Helquist, J. H., Santanen, E. L., \& Kruse, J. (2007). Participant-driven GSS: Quality of brainstorming and allocation of participant resources. 2007 40th Annual Hawaii International Conference on System Sciences (HICSS'07), 18-18. https://doi.org/10.1109/HICSS.2007.425

Hernandez, N. V., Shah, J. J., \& Smith, S. M. (2010). Understanding design ideation mechanisms through multilevel aligned empirical studies. Design Studies, 31(4), 382-410. https://doi.org/10.1016/j.destud.2010.04.001 Holper, L., ten Brincke, R. H. W., Wolf, M., \& Murphy, R. O. (2014). FNIRS derived hemodynamic signals and electrodermal responses in a sequential risk-taking task. Brain Research, 1557, $141-154$. https://doi.org/10.1016/j.brainres.2014.02.013

Howard, T. J., Culley, S. J., \& Dekoninck, E. (2008). Describing the creative design process by the integration of engineering design and cognitive psychology literature. Design Studies, 29(2), 160-180. https://doi.org/10.1016/j.destud.2008.01.001

Hu, M., \& Shealy, T. (2019). Application of functional Near-Infrared Spectroscopy to measure engineering decision-making and design cognition: Literature review and synthesis of methods. Journal of Computing in Civil Engineering, 33(6), 04019034. https://doi.org/10.1061/(ASCE)CP.1943-5487.0000848

Isaksen, S. G., \& Gaulin, J. P. (2005). A reexamination of brainstorming research: Implications for research and practice. Gifted Child Quarterly, 49(4), 315-329. https://doi.org/10.1177/001698620504900405

Kovacevic, N., Ritter, P., Tays, W., Moreno, S., \& McIntosh, A. R. (2015). 'My Virtual Dream': Collective neurofeedback in an immersive art environment. PLOS ONE, 10(7). https://doi.org/10.1371/journal.pone.0130129 
Osborn, A. F. (1953). Applied Imagination: Principles and Procedures of Creative Thinking. Creative Education Foundation, Buffalo, NY.

Pinsonneault, A., Barki, H., Gallupe, R. B., \& Hoppen, N. (1999). Electronic brainstorming: The illusion of productivity. Information Systems Research, 10(2), 110-133.

Pochon, J. B., Levy, R., Fossati, P., Lehericy, S., Poline, J. B., Pillon, B., Dubois, B. (2002). The neural system that bridges reward and cognition in humans: An fMRI study. Proceedings of the National Academy of Sciences of the United States of America, 99(8), 5669-5674. https://doi.org/10.1073/pnas.082111099

Potter, R., \& Balthazard, P. (2004). The role of individual memory and attention processes during electronic brainstorming. Management Information Systems Quarterly, 28(4).

Rosen, D. S., Erickson, B., Kim, Y. E., Mirman, D., Hamilton, R. H., \& Kounios, J. (2016). Anodal tDCS to right dorsolateral prefrontal cortex facilitates performance for novice jazz improvisers but hinders experts. Frontiers in Human Neuroscience, 10. https://doi.org/10.3389/fnhum.2016.00579

Shah, J. J., Smith, S. M., \& Vargas-Hernandez, N. (2003). Metrics for measuring ideation effectiveness. Design Studies, 24(2), 111-134. https://doi.org/10.1016/S0142-694X(02)00034-0

Shealy, T., \& Gero, J. (2019). The neurocognition of three engineering concept generation techniques. Proceedings of the Design Society: International Conference on Engineering Design, 1(1), 1833-1842. https://doi.org/10.1017/dsi.2019.189

Shealy, T., Grohs, J., Hu, M., Maczka, D., \& Panneton, R. (2017). Investigating design cognition during brainstorming tasks with freshmen and senior engineering students using functional Near Infrared Spectroscopy. ASEE Annual Conference, Columbus, OH.

Shealy, T., \& Hu, M. (2017). Evaluating the potential of neuroimaging methods to study engineering cognition and project-level decision making. Presented at the EPOC-MW Conference, Engineering Project Organization Society, Fallen Leaf Lake, CA USA.

Shealy, T., Hu, M., \& Gero, J. (2018). Patterns of cortical activation when using concept generation techniques of brainstorming, morphological analysis, and TRIZ. ASME 2018 IDETC/CIE, Quebec city, Canada.

Smith, G. F. (1998). Idea-Generation techniques: A formulary of active ingredients. The Journal of Creative Behavior, 32(2), 107-134. https://doi.org/10.1002/j.2162-6057.1998.tb00810.x

Soltanlou, M., Sitnikova, M. A., Nuerk, H.-C., \& Dresler, T. (2018). Applications of functional Near-Infrared Spectroscopy (fNIRS) in studying cognitive development: The case of mathematics and language. Frontiers in Psychology, 9. https://doi.org/10.3389/fpsyg.2018.00277

Storm, B. C., \& Hickman, M. L. (2015). Mental fixation and metacognitive predictions of insight in creative problem solving. Quarterly Journal of Experimental Psychology, 68(4), 802-813. https://doi.org/10.1080/17470218.2014.966730

Tilahun, N., Thakuriah, P., Li, M., \& Keita, Y. (2016). Transit use and the work commute: Analyzing the role of last mile issues. Journal of Transport Geography, 54, 359-368. https://doi.org/10.1016/j.jtrangeo.2016.06.021

Viswanathan, V. K. (2017). Design fixation to examples: A study on the time decay of fixation. Proceedings of the 21st International Conference on Engineering Design (ICED 17) Vol 8: Human Behaviour in Design, Vancouver, Canada.

Zhang, M., Liu, T., Pelowski, M., \& Yu, D. (2017). Gender difference in spontaneous deception: A hyperscanning study using functional near-infrared spectroscopy. Scientific Reports, 7(1), 7508. https://doi.org/10.1038/s41598017-06764-1 